\title{
Control de la calidad del diagnóstico coproparasitológico en la provincia de Ciudad de La Habana, Cuba
}

\author{
External quality assessment in coproparasitology \\ in Havana City Province, Cuba
}

Fidel Angel Núñez 1

Dora E. Ginorio 2

Carlos M. Finlay 1

\footnotetext{
1 Instituto de Medicina Tropical "Pedro Kourí", Apartado postal 601, Ciudad Habana, Cuba 2 Centro Provincial de Higiene, Epidemiología y Microbiología (CPHEM) de Ciudad Habana, Ministerio de Salud Pública, Ciudad Habana, Cuba.
}

Abstract An external quality assessment in coproparasitology was carried out in 77 laboratories from Havana City. A questionnaire and ten plastic vials with different intestinal parasites in a small nyl on bag, duly sealed, were sent to each laboratory. Answers were collected during the 72 hours after delivery. Results were analyzed by means of a computer program. The maj ority of the laboratories (70\%) passed the test; the municipalities with the worst scores in the province were Lisa, Marianao, and Habana del Este. Better results were obtained among technologists working only in parasitology than those who were also performing other laboratory work, and better averages were observed in hospitals than in polyclinics. The best identified intestinal protozoan was Giardia lamblia and the worst identified was Blastocystis hominis (with a $61 \%$ mistake rate), followed by Endolimax nana (24.6\%), and Entamoeba histolytica (22\%). Among hel minths, the best identified was Trichuris trichiura (9.2\% mistake rate) and the highest percentage of incorrect diagnoses was for Taenia sp. and Fasciola hepatica (both with 66.2\%). Taking into account these results, we feel it is necessary to provide training in parasitology among these laboratories.

Key words Quality Control; Diagnosis; Parasitology; Coproparasitology

Resumen Se realizó un estudio sobre la calidad del diagnóstico coproparasitológico en 77 laboratorios dela red de salud pública de la provincia Ciudad de La Habana, Cuba. El procedimiento se basó en la entrega a cada jefe de laboratorio de un modelo de encuesta, y una bolsa de nylon conteniendo 10 viales plásticos con distintos especímenes parasitarios, preservados en formaldehído al 7\%. Recogi dos los resultados en las primeras 72 horas después de su entrega, se realizó la evaluación mediante una escala de puntuación estableci da. La mayoría de los laboratorios aprobaron (70\%); sin embargo aún existen centros, sobre todo policlínicas, con calificaciones deficientes. Los munici pi os con resultados más desfavorables fueron, Lisa, Marianao y Habana del Este, al canzándose mejores resultados en los hospitales que en las policlínicas. En el análisis de Protozooarios, el mejor diagnosticado fué Giardia lamblia, con solo un centro que erró al identificarlo. Las mayores dificultades se presentaron en Blastocystis hominis con $61 \%$ de fallas, Endolimax nana, con 24,6\%, y Entamoeba histolytica, con $22 \%$. Entre los hel mintos, la mayor aprobación fué en Trichuris trichiura y los errores diagnósticos predominaron con Fasciola hepatica y Taenia sp., ambos con $66,2 \%$ de fallas. Dados Ios resultados obtenidos, hemos organizado una intervención educativa en la red delaboratorios de la provincia.

Palabras clave Control de Calidad; Diagnóstico; Parasitología, Coproparasitología 


\section{Introducción}

El diagnóstico de la mayoría de las infecciones por parásitos intestinales se fundamenta aún en el examen de material fecal, dado que se han desarrollado pocas técnicas coproparasitológicas en los últimos 50 años (WHO, 1987), como las de coprocultivo de larvas (Harada \& Mori, 1955) y, el frotis grueso de Kato \& Miura (1954), modificado por Katz et al. (1972).

A pesar de existir una tendencia en los últimos años a la aplicación de técnicas de inmunodiagnóstico, como la detección de antígenos en heces por medio de un inmunoensayo enzimático (Del Muro et al., 1987; Bauman \& Gottstein, 1987; Merino et al., 1990), estas son más aplicables en la investigación y no en la práctica diaria del diagnóstico en los laboratorios clínicos, pues la relación costo-beneficio no justifica su empleo (WHO, 1992). Además, las técnicas de diagnóstico coproparasitológico son las más utilizadas en programas de control del parasitismo intestinal por su bajo costo, simplicidad y sensibilidad.

En nuestro país se realiza el diagnóstico coproparasitológico en la red de salud por los métodos tradicionales deWillis y el examen directo (Grupo Nacional de Laboratorios Clínicos, 1969). En hospitales pediátricos se han extendido otros métodos diagnósticos como el de Ritchie (Ash \& Orihel, 1987), y el de Zielh Neelsen modificado para Cryptosporidium (Henriksen \& Pohlenz, 1981). Sin embargo existen algunos problemas de diagnóstico en la red de salud, como errores por sobrediagnóstico en el caso de Entamoeba histolytica, confundida con macrófagos y piocitos. Este último problema no solo es nacional, pues ha sido reportado en varios países, cada vez con mayor énfasis (Krogstad et al., 1978; Anaya-Velazquez \& Sabanero-López, 1989).

El siguiente estudio tiene como objetivos evaluar la calidad del diagnóstico coproparasitológico en unidades de la red de salud en la provincia Ciudad de La Habana, determinar cuales son los problemas que pueden afectar el diagnóstico de las parasitosis intestinales y establecer los factores que pueden estar incidiendo en estos.
Materiales y métodos

Se elaboró un modelo de encuesta que contenía diferentes datos de interés para nuestro estudio. Entre los meses de M ayo y Septiembre de 1993 se entregaron a cada centro esta planiIla y una bolsa de nylon numerada conteniendo 10 viales plásticos, uno de ellos con heces negativas y el resto con los especímenes siguientes: huevos de Trichuris trichiura, Ascaris I umbricoides, Taenia sp., y Fasciola hepatica; quistes de Entamoeba histolytica, Giardia lamblia, Endolimax nana, y Entamoeba coli, y formas anulares de Blastocystis hominis; todos debidamente preservados en solución de formaldehído al 7\%

Los parásitos se obtuvieron a partir de muestras positivas de heces analizadas en nuestros laboratorios, con especímenes en cantidades suficientes (4 ó más parásitos por campo microscópico a $400 \mathrm{X}$ de aumento), con excepción de Taenia sp., donde se obtuvieron huevos a partir de proglótides grávidos macerados y mezclados en forma homogénea con heces negativas y de F. hepatica donde se emplearon huevos obtenidos de bilis de ganado bovino infectado, que fueron mezclados de igual forma. Las heces negativas fueron procesadas por varias técnicas coproparasitológicas (Directo, Ritchie, Faust, y Willis), y observadas diariamente durante 15 días por 3 técnicos, para garantizar su condición negativa. Además, fueron confeccionados juegos de viales extras en cada lote de preparación, para comprobar su conservación al observarlos 3 y 6 meses después de preparados.

Los números que identificaban los viales se cambiaron según el centro asistencial al que se destinó la muestra y las encuestas fueron recogidos dentro de las 72 horas posteriores a la entrega.

Para clasificar los resultados obtenidos se consideró una calificación de 10 puntos: excelente; 9 puntos: muy bien; 8 puntos: bien; 7 puntos: regular; y menos de 7 puntos: mal. Para esto se tuvo en cuenta que sólo se utilizaron parásitos autóctonos, en alta concentración y que el técnico que realizaba el diagnóstico era seleccionado por el jefe del laboratorio, pudiendo participar en la identificación más de un técnico, lo cual conduciría a la evaluación de la capacidad diagnóstica del laboratorio.

\section{Análisis Estadístico}

Los resultados fueron procesados con una base de datos DBASE III plus en una microcomputadora personal IBM AT. Se emplearon los estadígrafos de comparación de proporciones para 
analizar la diferencia entre los grupos. Se aceptó como nivel de significación un valor de $p<$ 0,05 . Para realizar la comparación de medias se empleó la prueba " $\mathrm{t}$ " de Student.

\section{Resultados}

En la tabla 1 se agrupan las medias y desviaciones estándares de las calificaciones en base a 10 puntos que obtuvieron los diferentes municipios de Ciudad Habana, excepto Regla, San Miguel del Padrón y Boyeros, con solo un centro encuestado, cifra que hace imposible el empleo de estas medidas de tendencia central para nuestro análisis. Los municipios de Regla y San Miguel del Padrón presentaron los mejores resultados, con una calificación de 9 puntos, y Centro Habana obtuvo una media de 8 puntos. Los municipios más desfavorables fueron Marianao, con 6,5 puntos, seguido por Lisa y $\mathrm{Ha}$ bana del Este, ambos con 5 puntos.

En la tabla 2, se emplea el estadígrafo de comparación de proporciones y se compara el porciento de aprobación del control de calidad entre los técnicos fijos en la sección de Parasitología, con los que trabajan en forma rotatoria. Se observa que el mayor porciento de aprobados está en el primer grupo $(p \varangle 0,01)$, lo que demuestra el mejor diagnóstico parasitológico.

La tabla 3 muestra mejores calificaciones en los hospitales que en las policlínicas, siendo la media de la puntuación de los hospitales mayor que la de las policlínicas (Prueba " $\mathrm{t}$ " de Student, $p \varangle 0,01$ ).

Al analizar las fallas diagnósticas cometidas por los laboratorios en la identificación de especies de protozoos, observamos que G. Iamblia presentó un índice de diagnósticos satisfactorios, al fallar sólo un centro (1,3\%). Mientras que las mayores dificultades en la identificación fueron con $\mathrm{B}$. hominis, donde el $61 \%$ de los centros no lo diagnosticaron, seguido de E. nana, con $24,6 \%$ de errores, y E. histolytica, con $22 \%$ (Tabla 4).

Dentro de los helmintos, el mejor identificado fué T. trichiura, y los mayores equívocos fueron para Taenia sp. y F. hepatica, donde el $66,2 \%$ de los centros no consiguieron su identificación (Tabla 5).

Otros aspectos deficientes encontrados en más del $50 \%$ de los centros encuestados son enumerados a continuación:

1) No se utilizan nombres científicos, empleán dose nombres vulgares para informar los resultados. Ejemplo: tricocéfalos en vez de T. trichiura.

2) No se realiza, al menos, un método de con- centración (Willis), usándose sólo el examen directo.

3) Se emplea para el examen coprológico microscopios defectuosos, o de deficiente visión. 4) Los laboratorios no poseen muestras positivas para referencia.

5) No existe un control interno, ni externo de la calidad del diagnóstico parasitológico.

Tabla 1

Resultados de la evaluación en 13 municipios de Ciudad de La Habana.

\begin{tabular}{lccc}
\hline M unicipio & No de Laboratorios & \multicolumn{2}{c}{$\begin{array}{c}\text { Calificación } \\
( \pm D S)\end{array}$} \\
\hline Regla & 1 & $9,0 *$ & - \\
San M. del Padrón & 1 & $8,0 *$ & - \\
Centro Habana & 8 & 8,0 & $( \pm 1,41)$ \\
Plaza & 11 & 7,6 & $( \pm 1,61)$ \\
Cerro & 7 & 7,7 & $( \pm 1,16)$ \\
Playa & 7 & 7,4 & $( \pm 1,04)$ \\
Habana Vieja & 5 & 7,2 & $( \pm 0,97)$ \\
Boyeros & 1 & $7,0 *$ & - \\
10 de O ctubre & 11 & 7,0 & $( \pm 1,53)$ \\
Arroyo Naranjo & 8 & 7,0 & $( \pm 0,86)$ \\
Marianao & 6 & 6,5 & $( \pm 1,70)$ \\
Lisa & 6 & 5,0 & $( \pm 1,29)$ \\
Habana del Este & 5 & 5,0 & $( \pm 1,00)$ \\
Total & 77 & 7,0 & $( \pm 1,59)$ \\
\hline
\end{tabular}

* Valor puntual

Tabla 2

Resultados de la evaluación según la permanencia en la sección de Parasitología.

\begin{tabular}{|c|c|c|c|c|}
\hline \multirow[t]{3}{*}{ Resultados de la Evaluación } & \multicolumn{4}{|c|}{ Permanencia en la sección } \\
\hline & \multicolumn{2}{|c|}{ Fijos } & \multicolumn{2}{|c|}{ Rotatorios } \\
\hline & $\mathrm{n}$ & $\%$ & $\mathrm{n}$ & $\%$ \\
\hline Aprobados (7 - 10 puntos) & 6 & 66,6 & 21 & 30,8 \\
\hline Desaprobados (menos de 7) & 3 & 33,3 & 47 & 69,1 \\
\hline Total & 9 & & 68 & \\
\hline
\end{tabular}

$p<0,01$

Tabla 3

Resultados obtenidos según el tipo de unidad asistencial.

\begin{tabular}{lcc}
\hline Tipo de Unidad & \multicolumn{2}{c}{ Calificación } \\
& $x$ & $( \pm D S)$ \\
\hline Hospitales $(n=22)$ & 8,0 & $( \pm 1,29)$ \\
Policlínicas $(n=55)$ & 6,5 & $( \pm 1,49)$ \\
Total $(n=77)$ & 7,0 & $( \pm 1,59)$
\end{tabular}

Prueba " $t$ " de Student, $p<0,01$. 
Tabla 4

Fallas en la identificación de especies de protozoos en los laboratorios.

\begin{tabular}{lcc}
\hline \multirow{2}{*}{ Protozoo sin Identificar } & \multicolumn{2}{c}{ Unidades } \\
& $\mathrm{n}$ & $\%$ \\
\hline Giardia lamblia & 1 & 1,3 \\
Entamoeba coli & 6 & 7,8 \\
Entamoeba histolytica & 17 & 22,0 \\
Endolimax nana & 19 & 24,6 \\
Blastocystis hominis & 47 & 61,0 \\
Total & 77 & \\
\hline
\end{tabular}

Tabla 5

Fallas en la identificación de helmintos en los laboratorios de la capital.

\begin{tabular}{lcc}
\hline \multirow{2}{*}{ Helminto sin Identificar } & \multicolumn{2}{c}{ Unidades } \\
& $\mathrm{n}$ & $\%$ \\
\hline Trichuris trichiura & 7 & 9,1 \\
Ascaris lumbricoides & 17 & 22,0 \\
Fasciola hepatica & 51 & 66,2 \\
Taenia sp. & 51 & 66,2 \\
Total & 77 & \\
\hline
\end{tabular}

\section{Discusión}

El control de calidad en Parasitología no ha sido difundido lo suficiente como en otras ramas del diagnóstico del laboratorio clínico y fue incorporado en la práctica de la salud pública sólo en los últimos años. En los países desarrollados comenzó a emplearse de forma sistemática a finales de los años 70. En Francia, comenzó en Junio de 1977 (Petithory, 1979), casi al mismo tiempo que la encuesta de calidad en Parasitología del Colegio de Patólogos Americanos (Smith, 1979), y, en Gran Bretaña, comenzó en 1986 (Hawthorne et al., 1992). En muchos países del mundo aún no se realizan controles para esta actividad; en América Latina se han realizado escasos y aislados estudios sobre el control de la calidad en coproparasitología (Ayala \& Sánchez, 1974). En Cuba, esta actividad comenzó en nuestro laboratorio a partir de 1992, cuando se desarrolló un estudio integral sobre la calidad del diagnóstico coproparasitológico en la provincia de Camagüey (Del Risco, 1992). Los resultados que se obtuvieron en ese estudio hicieron posible que se extendiera a otras provincias, destacándose la capital como una de las más importantes, por presentar la más alta densidad poblacional del país y la mayor red de laboratorios que brindan diagnóstico coproparasitológico.

Los resultados obtenidos en los diferentes municipios de la capital demuestran que, en general, la mayoría de los centros (70\%) aprobaron, al obtener calificaciones iguales o superiores a 7 puntos. Sin embargo, dentro de los municipios existen centros, sobretodo en las policlínicas, desaprobados. Las notas obtenidas en los municipios Lisa, Marianao y Habana del Este nos indican la necesidad evidente de emprender planes para readiestramiento en los mismos.

Los técnicos que trabajaban fijos en la sección de Parasitología obtuvieron mejores resultados que los que rotaban habitualmente. En estudio realizado por Mohr \& Mohr, (1992), se encontró una calidad superior del diagnóstico parasitológico en los tecnólogos que trabajaban solamente con Parasitología, que en los microbiólogos generales que también realizaban otros trabajos dentro del laboratorio. El nivel de especialización es una característica relevante para la calidad del diagnóstico coproparasitológico, por lo que, siempre que sea posible, se debe tratar de tener un personal fijo en la sección de Parasitología. Sin embargo, en las policlínicas, muchas veces es necesario que el técnico, para cumplir su jornada de trabajo, pase parte del tiempo en otras actividades del laboratorio; en estos casos ha de ser importante que este personal cumpla con ciclos de readiestramientos sistemáticos que lo capaciten para mantener un diagnóstico eficaz.

En cuanto a la calidad del diagnóstico coproparasitológico, los resultados fueron más desfavorables en los diferentes tipos de centros de atención primaria (policlínicas) que en los de atención secundaria y terciaria (hospitales).Esto puede explicarse porque, en los hospitales, se encuentran los laboratorios microbiológicos con una sección de Parasitología, donde se concentran casi todos los técnicos que están fijos en la sección. En ellos se han incorporado médicos microbiólogos con un perfil de especialización en Parasitología Clínica, lo que fortalece la calidad del diagnóstico. Mientras tanto, en las policlínicas no existen laboratorios microbiológicos, sino laboratorios clínicos donde la Parasitología se hace de forma rotatoria, con un ciclo de rotación corto y un tiempo breve de permanencia de los técnicos en la sección.

Dentro de los protozoos, el mejor identificado fué G. Iamblia, el que ha sido señalado en nuestro país como el más prevalente dentro de 
los protozoos patógenos (Núñez et al., 1989). El que mayores dificultades diagnósticas presentó fué B. hominis, con un alto porcentaje de fallas, hecho preocupante, pues, en los últimos años, este parásito, que era considerado anteriormente como levadura, ha ganado una importancia cada vez mayor como patógeno humano (Zierdt, 1991; Ashford \& Atkinson, 1992). En orden de frecuencia, le siguieron E. nana y E. histolytica; esta última es de gran importancia por su confusión diagnóstica con Entamoeba hartmanni (González-Ruiz \& Bendall, 1995), leucocitos y macrófagos (Healy, 1986).

Dentro de los helmintos, T. trichiura, el más prevalente en Cuba (Núñez et al., 1993), fué el mejor diagnosticado; mientras que los mayores problemas de identificación fueron para Taenia sp. y F. hepatica. Esto resulta paradójico, si se tiene en cuenta que el huevo de F. hepatica (hasta 150 micras de longitud) es el más grande de los huevos de los hel mintos intestinales autóctonos de nuestro país (Kourí et al., 1982). En el caso de Taenia sp., muchos laboratorios llegaron al diagnóstico de especie, como Taenia saginata, teniendo como elemento diagnóstico sólo los huevos, sin aplicar coloracio- nes especiales, lo cual es erróneo, pues los huevos de todas las especies de Taenia son idénticos en su morfología (Ash \& Orihel, 1987).

En este trabajo, la participación de los laboratorios fué voluntaria, pero pensamos que, en controles futuros, ésta debe ser de manera obligatoria. En Francia, aunque el control de calidad comenzó en forma voluntaria en 1977, se instauró en forma obligatoria y con un carácter legal a finales de 1979 (Pethitory, 1988). Sin embargo, la esencia del control no consiste en sancionar al técnico que realiza un mal diagnóstico, sino en evaluar este, al comparar sus resultados con los de otros laboratorios, constituyendo la forma más satisfactoria de realizar este trabajo con la debida fiabilidad y eficiencia (Pethitory, 1991). En los casos necesarios, llevar a cabo un readiestramiento o entrenamiento de los técnicos para lograr un diagnóstico con la calidad requerida.

Con los resultados obtenidos en este estudio, hemos diseñado un plan de intervención educativa en los laboratorios de la red de salud pública de la capital que, una vez ejecutado, se procederá a otra evaluación con vistas a medir sus efectos.

\section{Referencias}

ANAYA-VELAZQUEZ, F. \& SABANERO-LOPEZ, G., 1989. Use of Wright's stain to identify Entamoeba histolytica trophozoites in faeces. Transactions of the Royal Society of Tropical Medicine and Hygiene, 83:210.

ASH, L. R. \& ORIHEL, T. C., 1987. Parasites: A Guide to Laboratory Procedures and Identification. Chicago: ASCP Press.

ASHFORD, R. W. \& ATKINSON, E. A., 1992. Epidemiology of Blastocystis hominis infection in Papua New Guinea: age prevalence and associations with others parasites. Annals of Tropical Medicine \& Parasitology, 86:129-136.

AYALA, S. C. \& SANCHEZ, C. E., 1974. Evaluación de los diagnósticos coproparasitológicos de la ciudad de Cali. Acta Médica deValle, 5:114-121.

BAUM ANN, D. \& GOTTSTEIN, B., 1987. A double-antibody sandwich ELISA for the detection of Entamoeba histolytica antigen in stool samples of humans. Tropical Medicine and Parasitology, 38:8185.

DEL MURO, R.; OLIVA, A.; HERION, P.; CAPIN, R. \& ORTIZ-ORTIZ, L., 1987. Diagnosis of Entamoeba histolytica in feces by ELISA. Journal of Clinical Laboratory Analysis, 1:322-325.
DEL RISCO, U., 1992. Control dela Calidad del Diagnóstico Coproparasitológico en la Provincia deCamagüey. Tesis para especialista de primer grado en Microbiología. Ciudad de La Habana: Instituto "Pedro Kourí". Instituto Superior de Ciencias Médicas de La Habana.

GONZALEZ-RUIZ, A. \& BENDALL, R. L., 1995. Size Matters: The use of ocular micrometer in Diagnostic Parasitology. Parasitology Today, 11:83-85.

GRUPO NACIONAL DE LABORATORIOS CLINICOS, MINSAP., 1969. Manual de Técnicas para Laboratorios Clínicos. La Habana: Ciencia y Técnica.

HARADA, Y. \& MORI, O., 1955. A new method for culturing hookworm. Yonago Acta Medica, 1:177-179.

HAWTHORNE, M.; CHIODINI, P. L.; SNELL, J. J.; MOODY, A. H. \& RAM SEY, A., 1992. Parasitology Quality Assessment, United Kingdom National Scheme. Journal of Clinical Pathology, 45:968974.

HEALY, G. R., 1986. Immunologic tools in the diagnosis of amebiasis: epidemiology in the United States. Reviews of Infectious Diseases, 8:239-245.

HENRIKSEN, S. A. \& POHLENZ, J. F. L., 1981. Staining of cryptosporidia by a modified Ziehl-Neelsen technique. Acta Veterinarica Scandinavica, 22: 594-596. 
KATO, K. \& MIURA, M., 1954. Comparative Examinations. (japanese text). Japan Journal of Parasitology, 3:35.

KATZ, N.; CHAVEZ, A. \& PELLEGRINO, J., 1972. A simple device for quantitative stool thick-smear technique in schistosomiasis mansoni. Revista do Instituto de Medicina Tropical de São Paulo, 14: 397-400.

KOURI, P.; BASNUEVO, J. G. \& SOTOLONGO, F., 1982. Helmintología Humana. Ciudad Habana: Editorial Pueblo y Educación.

KROGSTAD, D. J.; SPENCER, H. C.; HEALY, D. R.; GLEASON, N. N., SEXTON, D. J. \& HERION, C. A., 1978. Amebiasis: Epidemiologic studies in the United States, 1971-1974. Annals of Internal Medicine, 88:89-97.

MERINO, E.; GLENDER, W.; DEL MURO, R. \& ORTIZORTIZ, L., 1990. Evaluation of the ELISA test for detection of Entamoeba histolytica in feces. Journal of Clinical Laboratory Analysis, 4:39-42.

MOHR, E. \& MOHR, I., 1992. Statistical analysis of the incidence of positives in the examination of parasitological specimens. Journal of Clinical Microbiology, 30:1572-1574.

NÚÑEZ, F. A.; SANJURJO, E. \& FINLAY, C. M., 1989. Estudio de la Giardiasis en una comunidad rural. Revista de la Asociación Guatemalteca de Parasitología y Medicina Tropical, 4:13-18.
NÚÑEZ, F. A.; SANJURJO, E.; BRAVO, J. R.; CARBALLO, D. \& FINLAY, C. M., 1993. Trichuriasis en Cuba. Revista Cubana de Medicina Tropical, 45: 42-45.

PETITHORY, J. C., 1979. Le contrôle de qualité en parasitologie. Bulletin de la Société de Pathologie Exotique, 72:386-395.

PETITHORY, J. C., 1988. Organization and interest of external quality assessment in parasitology. Arabian Journal of Laboratory Medicine, 14:145-156.

PETITHORY, J. C., 1991. Bilan de dix ans de contrôle national de qualité en parasitologie. Cahiers Techniques de la Biologie, 24:3-9.

SMITH, J. W., 1979. Identification of fecal parasites in the special parasitology survey of the College of American Pathologists. American Society of Clinical Pathology, 72:371-373.

WHO (World Health Organization), 1987. Prevention and Control of Intestinal Parasitic Infections. Geneve: WHO, Expert Committee. (Technical Report Series, 749).

WHO (World Health Organization), 1992. Informal Consultation on Intestinal Protozoal Infections. Mexico: WHO/PAHO (WHO/CDS/IPI/ 92.2.)

ZIERDT, C. H., 1991. Blastocystis hominis - past and future. Clinical Microbiology Reviews, 4:61-79. 